Relations industrielles

Industrial Relations

\title{
Les attitudes des travailleurs québécois à l'égard de leur emploi, par Louise H. Côté-Desbiolles, Québec, ministère du Travail et de la Main-d'oeuvre, collection « études et recherche ", 1979, 145 pp.
}

\section{Alain Vinet}

Volume 35, numéro 2, 1980

URI : https://id.erudit.org/iderudit/029072ar

DOI : https://doi.org/10.7202/029072ar

Aller au sommaire du numéro

\section{Éditeur(s)}

Département des relations industrielles de l'Université Laval

ISSN

0034-379X (imprimé)

1703-8138 (numérique)

Découvrir la revue

Citer ce compte rendu

Vinet, A. (1980). Compte rendu de [Les attitudes des travailleurs québécois à l'égard de leur emploi, par Louise H. Côté-Desbiolles, Québec, ministère du Travail et de la Main-d'oeuvre, collection « études et recherche ", 1979, 145 pp.] Relations industrielles / Industrial Relations, 35(2), 341-342.

https://doi.org/10.7202/029072ar

Tous droits réservés (C) Département des relations industrielles de l'Université Laval, 1980
Ce document est protégé par la loi sur le droit d'auteur. L'utilisation des services d'Érudit (y compris la reproduction) est assujettie à sa politique d'utilisation que vous pouvez consulter en ligne.

https://apropos.erudit.org/fr/usagers/politique-dutilisation/ 
sonnelles et organisationnelles dans l'industrie, la conception traditionnelle des relations industrielles n'a cerné, selon Margerison, que la moitié du problème...» (p. 74). Nous servant de cette citation comme d'un point de référence, nous compléterons le commentaire en soulignant que l'auteur, comme on pouvait s'y attendre, allait non seulement accorder beaucoup d'attention aux multiples causes et aspects des conflits du travail mais allait aussi, du même coup, passer en revue les contributions des différentes sciences sociales et notamment la sociologie et les sciences du comportement - en vue de la prévention ou de la solution de ces conflits, qu'ils soient «interpersonnels» ou "collectifs», les premiers pouvant d'ailleurs amener les seconds - et la solution apportée aux premiers pouvant ainsi empêcher les seconds. Dans ce livre (comme dans ses autres), l'auteur continue à accorder une importance, à notre avis très utile, à ces clarificateurs par excellence que sont les définitions: par exemple, il situe et définit la notion même de relations industrielles (pp. 3-11) et celle de «conflits collectifs du travail» (pp. 86-91). Par ailleurs, il traite d'une façon particulièrement détaillée de l'absentéisme et de ses causes et fournit sur le sujet de précieuses (et actuelles) informations. (Voir «Une Conflictualité latente: la désaffection au travail» pp. IX-X). Sous le titre "Les Remèdes aux dysfonctionnements dans le travail» (pp. 155-210), l'auteur offre faits et idées qui intéresseront non seulement les étudiants des relations industrielles mais aussi celles et ceux dont c'est le métier de prévenir et d'aplanir les conflits du travail, quelles qu'en soient l'origine et la nature.

Il faut souhaiter, dans l'intérêt de nos relations industrielles, que le livre de Dimitri Weiss, de portée internationale, soit bien accueilli non seulement dans nos institutions d'enseignement mais aussi parmi nos praticiens tant du secteur public que du secteur privé.

Félix QUINET

Bureau de Recherches sur

les Traitements,

Ottawa.
Les attitudes des travailleurs québécois à l'égard de leur emploi, par Louise $\mathrm{H}$. Côté-Desbiolles, Québec, ministère du Travail et de la Main-d'oeuvre, collection «études et recherches», 1979, 145 pp.

Ce rapport de recherche présente les résultats d'une vaste enquête sur la satisfaction au travail au Québec, effectué par le ministère du Travail et de la Main-d'oeuvre dans les premiers mois de l'année 1979. Cette étude s'inscrit tout à fait dans le cadre des enquêtes nationales amorcées sur le sujet dans plusieurs pays industrialisés depuis 1970. À peu de chose près, une même stratégie préside à l'élaboration de ces diverses enquêtes qui offrent par ailleurs des résultats très voisins.

Dans le cas présent, un questionnaire a été envoyé à 3911 personnes selon une procédure d'échantillonnage rigoureuse dans l'espoir d'obtenir 2000 réponses. L'échantillon utilisable de 2265 répondants s'est avéré représentatif (en terme de proportionnalité) de la population en emploi tant au niveau de l'âge, du sexe, du lieu de résidence, du secteur d'activité et de la catégorie d'emplois. Le nombre de questionnaires remplis a permis d'effectuer les croisements prévus dans les réponses des sujets. De façon générale, la méthode suivie répond aux règles en usage dans ce type d'enquête.

Le questionnaire utilisé pour cette étude combine deux formules fréquemment appliquées dans les enquêtes nationales: une évaluation de la satisfaction globale des répondants face à l'emploi est d'abord obtenue à l'aide de questions très générales; par la suite, les niveaux de satisfaction recueillis sont comparés avec des appréciations portant sur des aspects particuliers de l'emploi. Sept composantes de l'emploi sont ainsi examinées: le salaire, les possibilités d'avancement, l'intérêt du travail, les supérieurs, la sécurité d'emploi, les collègues de travail et les heures de travail. Pour chacune des composantes, l'enquête visait à mesurer la satisfaction de la personne mais aussi à connaître l'importance relative de cet élément aux yeux de l'informateur. Les résultats ont été analysés en fonc- 
tion de quatre caractéristiques des répondants: le sexe, l'âge, la catégorie d'emplois et le secteur d'activité économique. Les questions utilisées s'inspirent de plusieurs enquêtes étrangères et, dans l'ensemble, l'étude apparaît un modèle du genre.

Les résultats de ce type d'étude sont prévisibles et les Québécois ne font pas exception à la règle. L'auteur en est le premier conscient puisqu'elle présente les résultats de nombreuses autres études et s'interroge sur leur utilité. Dans le cas présent, les travailleurs québécois se disent très satisfaits ou assez satisfaits de leur emploi dans une proportion de $88 \%$. Ce haut niveau de satisfaction correspond à peu près parfaitement aux résultats obtenus en Australie, en Belgique, au Royaume Uni, aux États-Unis et au Canada. Une évaluation à l'aide de questions générales ne permet pas de déceler des variations dans la satisfaction au travail au cours des ans. Une répartition des réponses selon le sexe n'entraîne pas de différence significative. Seuls les jeunes travailleurs (24 ans et moins) sont moins nombreux à se dire très satisfaits ou assez satisfaits de leur emploi.

Cependant, les niveaux de satisfaction baissent sensiblement lorsque les enquêteurs utilisent des questions indirectes et davantage. orientées vers l'action. Ainsi seulement $67 \%$ des travailleurs accepteraient le même emploi et $63 \%$ le recommanderaient à un ami. Cette variation indique bien les difficultés méthodologiques de telles enquêtes et le peu de raffinement des indicateurs utilisés. On peut se demander ce que ces questions mesurent réellement: la satisfaction, l'adaptation des attentes à la réalité, la résignation, l'estime de soi, etc... En comparant les résultats de plusieurs enquêtes nationales, l'auteur souligne ces faiblesses et se défend de vouloir substituer des études d'opinion à l'analyse objective des conditions de travail. Les hauts niveaux de satisfaction obtenus régulièrement dans ces enquêtes imposent donc une réflexion à deux volets: remise en cause de la méthodologie et plus fondamentalement de l'utilité de ces enquêtes d'une part; mais aussi réflexion critique sur plusieurs analyses très intellectuelles du mécontentement des tra- vailleurs et sur les prédictions répétées d'affrontements spectaculaires qui les accompagnent. Bref, il y a place pour la recherche et la discussion.

L'aspect le plus intéressant de cette étude est sans contredit l'évaluation de la satisfaction des travailleurs à l'égard des composantes particulières de l'emploi. Dans cette partie de l'ouvrage, l'auteur of fre une analyse de chaque composante selon les quatre caractéristiques des répondants mentionnées précédemment. Cette analyse peut constituer dans certains cas le point de départ d'études ultérieures plus approfondies. Mentionnons que pour chacune des composantes, le niveau de satisfaction est généralement élevé bien que deux facteurs paraissent moins satisfaisants: le salaire et les possibilités d'avancement. Fait prévisible, les jeunes sont moins satisfaits de leurs perspectives de carrière, et la catégorie d'emploi du répondant est étroitement associée à son appréciation du salaire et de ses possibilités d'avancement. Par contre, l'examen de l'importance relative accordée par les répondants aux différentes composantes de l'emploi n' a pas donné de résultats probants. La diversité d'opinion est trop grande pour qu'il soit possible d'identifier l'un ou l'autre aspect comme prépondérant. Cependant l'intérêt du travail, la sécurité d'emploi et le salaire paraissent plus valorisés que les possibilités d'avancement, les supérieurs, les collègues de travail et les heures de travail.

En résumé, cette enquête est avantageusement comparable à celles qui ont été poursuivies jusqu'à présent dans plusieurs pays industrialisés. Le problème de la signification des résultats, de la validité et en définitive de l'utilité de ce genre d'enquête demeure cependant entier, surtout si on espère en tirer des statistiques nationales ou tenter des comparaisons avec d'autres pays. Par contre, certains indicateurs plus raffinés au niveau des composantes de l'emploi peuvent servir d'amorce à des recherches ultérieures.

Alain VINET

Université Laval 